\title{
Declive urbano, estrategias de revitalización y redes de actores: el peso de las trayectorias locales a través de los casos de estudio de Langreo y Avilés (España) ${ }^{1}$
}

\author{
José Prada-Trigo ${ }^{2}$
}

\begin{abstract}
RESUMEN
Las "trayectorias locales" y los sustratos territoriales existentes en cada lugar (redes, actores, capital humano) tienen un papel primordial en las estrategias de revitalización puestas en marcha en las ciudades que han sufrido una crisis. La manera en que los actores locales se relacionan y las iniciativas que toman para tratar de revitalizar estos espacios determinan no solo las decisiones que podrán tomarse en el futuro, sino la propia evolución demográfica, económica, ambiental y social de la ciudad. El artículo analiza, a partir de estas cuestiones, dos ciudades medias españolas en declive pertenecientes a una región de tradición industrial, Asturias. En ellas, el peso de los factores locales puede explicar en buena medida su desigual trayectoria tras la crisis de sus respectivas industrias. Para ello, tras una presentación de sus procesos de auge y declive, se analizan las estrategias de revitalización llevadas a cabo y se interpretan estas en base al papel de los actores locales.
\end{abstract}

Palabras clave: Declive urbano, estrategias de revitalización, actores locales, trayectorias locales, gobernanza.

\begin{abstract}
Local paths and existing substrates in each territory (networks, stakeholders, human and economic capital) have a major role in the revitalization strategies of shrinking cities. The way in which local stakeholders are related and the initiatives they take to try and revitalize declining cities determine not only the future decisions, but also the demographic, economic, environmental and social evolution of the city itself. This article analyzes, on the basis of these questions, two mid-size Spanish cities that are declining and are located in a former industrial region, Asturias. In these cities, the weight of local factors can largely explain their uneven evolutionary local paths after the crisis in their respective industries. To this end, and following a presentation of their respective boom and bust trajectories, the implemented revitalization strategies are analyzed and interpreted according to the local stakeholders role.
\end{abstract}

Key words: Urban decline, shrinkage, revitalization strategies, local actors, path dependence, governance.

\footnotetext{
1 Artículo recibido el 5 de octubre de 2012, aceptado el 26 de junio de 2013 y corregido el 2 de julio de 2013
}

\footnotetext{
2 Consejo Superior de Investigaciones Científicas (España). E-mail: pradatrigo@gmail.com
} 
En los inicios del siglo XXI el término shrinking city ${ }^{3}$ se ha expandido de tal manera que en la literatura científica hoy se habla de "global shrinkage» en los procesos de declive urbano (Fol y Cunnigham-Sabot, 2010: 360; Martínez-Fernández et al., 2012: 214). No obstante, las trayectorias seguidas por determinados países o regiones (zonas de antigua industrialización, áreas de fuerte emigración, espacios sometidos a intensas dinámicas de suburbanización) otorgan diferentes realidades a este fenómeno. En España, la mayoría de las ciudades que se encuentran en la actualidad en declive responden a modelos de ciudades de tradición industrial que sufrieron una importante crisis en la segunda mitad del siglo XX (Prada, 2011: 45; Sánchez et al., 2012: 322). Sin embargo, incluso dentro de esta tipología, las trayectorias locales muestran casos muy próximos en lo espacial que, a pesar de compartir un pasado común, han evolucionado de manera muy distinta en sus estrategias de revitalización. De esta forma, la dinámica demográfica, económica o social de estas ciudades ha sido muy diferente entre unas y otras, encontrándose la reversión del declive urbano en un estado más o menos avanzado.

Los objetivos principales de este artículo son tres: el primero de ellos es el análisis de la desigual recuperación de unos espacios y otros desde la perspectiva que ofrece la posibilidad de combinar los objetivos que persiguen las estrategias de revitalización puestas en práctica con el tipo de factores que se activan para ello. En segundo lugar, poner de manifiesto el peso de los actores locales en estas estrategias y su importancia para explicar estas trayectorias divergentes. Finalmente, destacar el rol central de la concertación entre estos actores y de la gobernanza urbana como poleas para incentivar medidas consensuadas capaces de abordar en mayor medida las complejas realidades de las ciudades. Para ello, se desarrolla un breve marco teórico que pone de relieve los principales argumentos de la investigación y algunos debates actuales en torno a estas cuestiones. A continuación se realiza una aproximación descriptiva a la trayectoria de los casos de estudio, utilizando

3 En adelante se traducirá como declive urbano. indicadores para reforzar la incidencia de los procesos de declive y desigual revitalización. Por último, se analizan las principales estrategias llevadas a cabo en base al esquema teórico presentado, terminándose con unas breves conclusiones.

La investigación presenta un enfoque analítico-interpretativo que combina metodologías cualitativas y cuantitativas. En primer lugar, se recurre a un amplio abanico de fuentes (estudios previos, documentos de planeamiento, noticias de la prensa local y regional) junto a estadísticas económicas, demográficas y sociales, con la intención de situar las trayectorias de estos espacios. Sin embargo, la principal fuente de información, utilizada como referencia para la interpretación de las estrategias de revitalización analizadas la constituye la realización de treinta entrevistas a actores locales. Estos pertenecen a diferentes ámbitos (político, económico, movimientos sociales) e instituciones (públicas, privadas, sindicatos, partidos políticos). Su aporte ha resultado clave para el desarrollo de esta investigación ${ }^{4}$.

\section{Declive urbano, estrategias de revitalización y trayectorias locales en ciudades de tradición minero-industrial: una aproximación a estos procesos}

El declive urbano (o urban shrinkage en la terminología internacional) no es un fenómeno nuevo, puesto que los procesos de crecimiento y crisis a lo largo de la historia han sido puestos de manifiesto por diferentes autores (Oswalt, 2006: 23; Oswalt \& Rienets, 2006: 14). El concepto de ciudad en declive se acuñó en el ámbito científico de Alemania Occidental a finales de los años setenta del siglo XX para referirse a aquellas ciudades que, tras un proceso de declive económico, mostraban pérdidas de población, como sucedía en la región alemana del Ruhr

\footnotetext{
4 El autor agradece su colaboración y sus contribuciones a los entrevistados (14 en Langreo y 16 en Avilés).
} 
(Grossmann et al., 2008: 78-79). Unos años después, la desindustrialización, envejecimiento demográfico, emigración y descenso del precio de la vivienda en el este de Alemania, que siguieron a la caída del muro de Berlín, mantuvieron la vigencia de esta temática. A finales del siglo $X X$ se produjo una expansión de los estudios declive urbano a nivel internacional, emergiendo nuevos aspectos ligados al mismo. Entre ellos destacan la crisis de la ciudad central y el crecimiento suburbano en EE.UU. que provocaron el Ilamado "efecto donut» en los centros urbanos (Lucy \& Phillips, 2000), el acusado envejecimiento demográfico en Japón y sus efectos sobre la población (Fuji, 2006), o la propia globalización de la economía y la competencia internacional entre ciudades, que puede encontrarse tanto en países desarrollados como en desarrollo, lo que permite a algunos autores hablar de «declive a escala global» (Fol \& Cunnigham-Sabot, 2010: 360).

Por lo tanto, desde su origen, el concepto de ciudad en declive se ha extendido en su vinculación a procesos de naturaleza diversa, que configuran unos modelos de declive propios para cada territorio. Una de las definiciones más extendidas hoy es aquella que describe las shrinking cities "as an urban area - a city, part of a city, an entire metropolitan area or a town - that has experienced population loss, economic downturn, employment decline and social problems as symptoms of a structural crisis» (Martínez-Fernández et al., 2012: 214). Las manifestaciones más evidentes de este proceso de declive serían una reducción y envejecimiento de la población como consecuencia de la caída de la natalidad y de la emigración hacia otros lugares; la disminución del empleo y el incremento del paro; el surgimiento de problemas sociales (pobreza, drogadicción, apatía); la reducción de los recursos públicos disponibles o problemas medioambientales y aparición de ruinas industriales como consecuencia del cierre de las empresas locales.

Mientras que en Latinoamérica, por su dinámica demográfica y su historia urbana, los casos de declive urbano son más reducidos y se encuentran sobre todo circunscritos a fenómenos de desurbanización en grandes metrópolis o de pérdida de competitividad en pequeñas ciudades poco dinámicas (Audirac
\& Arroyo, 2010: 9-13), en Europa la situación es distinta. Allí, a problemas generales, derivados de la reducción de la natalidad o del acusado envejecimiento de la población (Birg, 2006: 101) hay que añadir las consecuencias negativas que sufren determinados espacios que se vieron sometidos a la reconversión de sus industrias durante la segunda mitad del siglo XX que hasta entonces mostraban un fuerte dinamismo (Aydalot, 1987). En el caso de España, este hecho supuso el declive del Ilamado "Arco Atlántico», donde regiones como Asturias o País Vasco sufrieron las consecuencias negativas de su acusada especialización industrial, y el mayor dinamismo del «Eje Mediterráneo», que tomó el relevo del crecimiento (Prada, 2011: 44).

En este sentido, entre los debates actuales en torno a la cuestión del declive y la revitalización urbana (Martínez-Fernández et al., 2012) se encuentra la dualidad entre unas trayectorias comunes, marcadas por un contexto de cambio económico al que se vieron enfrentadas la mayoría de las ciudades europeas de tradición industrial, y una evolución diferenciada de las mismas, en la que cada espacio redefinió una estrategia de revitalización. De esta forma, las investigaciones sobre casos de estudio de ciudades en declive han permitido arrojar luz sobre la existencia de trayectorias locales concretas y sobre la importancia de los sustratos locales a la hora de explicar las diferencias entre espacios, en teoría similares y enfrentados a un escenario común, pero con una evolución desigual (Hamdouch et al., 2012: 5-6), cuestión en la que este artículo profundiza.

Así, en paralelo al declive urbano es necesario introducir la cuestión de la revitalización de las ciudades en declive. La predominancia de una "obsesión por el crecimiento» en la planificación urbana (Bontje, 2004: 14) y los efectos negativos del declive llevan a la respuesta de los gobiernos de las diferentes administraciones ante los procesos de declive. El objetivo, en este sentido, es doble: por un lado, terminar con el declive urbano y con sus efectos negativos, y por el otro, retomar un crecimiento similar al de otras áreas del país. Las medidas para lograr esto pueden interpretarse de maneras muy diferentes. En este artículo se ha decidido por una lectura novedosa, que combina los objetivos principales de las 
medidas (Méndez, 2010: 85) con los factores o recursos que se pueden utilizar, recuperar o activar para llevarlas a cabo (Musterd \& Murie, 2010: 20-29). En ella, las estrategias locales y el «sustrato de actores» presente en cada territorio son elementos claves.

Por un lado, desde el objetivo de un desarrollo territorial integrado, Ricardo Méndez (2010: 85 y ss.) diferencia entre aquellas estrategias que pueden dirigirse a: [i] el desarrollo de la economía y del empleo; [ii] la mejora de la gobernanza y de la gestión colectiva de la ciudad; [iii] la innovación social y económica; y [iv] la mejora de la calidad de vida, del medio ambiente y de la recuperación del patrimonio (Figura $\mathrm{N}^{\circ} 1$ ). Así, las primeras se vincularían a aspectos como la promoción de nuevos sectores y la formación de clusters económicos o la reconversión de los sectores tradicionales para favorecer la competitividad económica y la creación de empleo. Las segundas pueden relacionarse con la creación de redes, mediante el desarrollo de una cultura de la cooperación y de mecanismos de gobernanza. Esto, que puede lograrse a través de órganos de concertación, incentivos a la participación ciudadana o iniciativas supralocales de gobierno, se considera hoy un elemento clave en los procesos de desarrollo urbano (Kooiman, 2003). El tercer tipo de estrategias se vincularía al fortalecimiento de la estructura de innovación de un territorio mediante el impulso a centros tecnológicos, espacios para emprendedores y centros de empresas. También a través de la creación de vínculos entre la investigación y los sectores económicos o la promoción urbana (Scott, 2006: 2-3). Por último, el cuarto tipo de estrategias se relaciona tanto con medidas dirigidas a la mejora de los equipamientos urbanos y las condiciones de vida de los residentes, la renovación del stock de vivienda, como con la mejora del medio ambiente o la recuperación del patrimonio minero-industrial (Precedo et al., 2010: 6-9).

Sin embargo, para Sako Musterd y Alan Murie (2010: 20 y ss.), la posibilidad de incluir estos objetivos en las agendas de los gobiernos locales depende en buena medida de la existencia o activación de algunos factores o recursos que influyen en la capacidad de maniobra de los actores, entre los que destacan: [i] la existencia de los Ilamados classic or hard factors (factores clásicos); [ii] la presencia de clusters territoriales con capacidad de aglutinar empresas e instituciones; [iii] la existencia de redes locales y trayectorias de colaboración entre actores; y [iv] las llamadas soft conditions o atractivos urbanos (Figura $\mathrm{N}^{\circ} 1$ ). Así, en primer lugar es fundamental la disponibilidad de capital, fuerza de trabajo, ayudas públicas o infraestructuras que actúan como incentivos a la atracción de empresas o a la puesta en marcha de ideas innovadoras. Aunque este tipo de recursos ha perdido cierto peso a la hora de condicionar el desarrollo de un territorio, mantiene buena parte de su importancia (Musterd \& Murie, 2010: 21). En segundo lugar, la existencia de clusters es consecuencia de trayectorias urbanas en los que estos se han configurado, de manera que su propia evolución se interrelaciona con los cambios acaecidos en las estructuras socioeconómicas y en la propia ciudad. De esta forma, pueden vincularse a como determinadas ciudades son capaces de construir medios innovadores dando nuevas respuestas a los retos actuales (Camagni \& Maillat, 2006). En el caso de las ciudades de tradición industrial, la especialización en sectores tradicionales tuvo un papel fundamental en el pasado a la hora de explicar su crecimiento económico. En tercer lugar, destaca la naturaleza, densidad, composición y «masa crítica» de las redes de actores que se establecen en estas ciudades. El aumento de los retos a los que las ciudades se enfrentan en la actualidad hace necesaria la activación y el refuerzo de las redes de actores existentes, de forma que sean capaces de responder a un entorno crecientemente complejo (Pinson, 2009: 234 y ss.). Por último, las conocidas como soft conditions tienen un peso variable (y polémico) como cuarto factor. Este concepto, que acoge a un conjunto más o menos amplio de cuestiones relacionadas con los «atractivos urbanos», los «ambientes innovadores», el «grado de tolerancia» en una ciudad, constituyen un conjunto de aspectos subjetivos y flexibles que han tenido un gran éxito en los últimos años, popularizados en buena medida gracias al concepto de clase creativa (Florida, 2002). Desde esta perspectiva se considera a los trabajadores cualificados en los segmentos de la llamada economía creativa como las poleas de la recuperación de muchas ciudades. Sin embargo, las críticas que han sufrido las tesis de Florida (Peck, 2005; Simmie, 2005, entre 
Figura $\mathrm{N}^{0} 1$

Perspectiva interpretativa de los procesos de revitalización urbana.

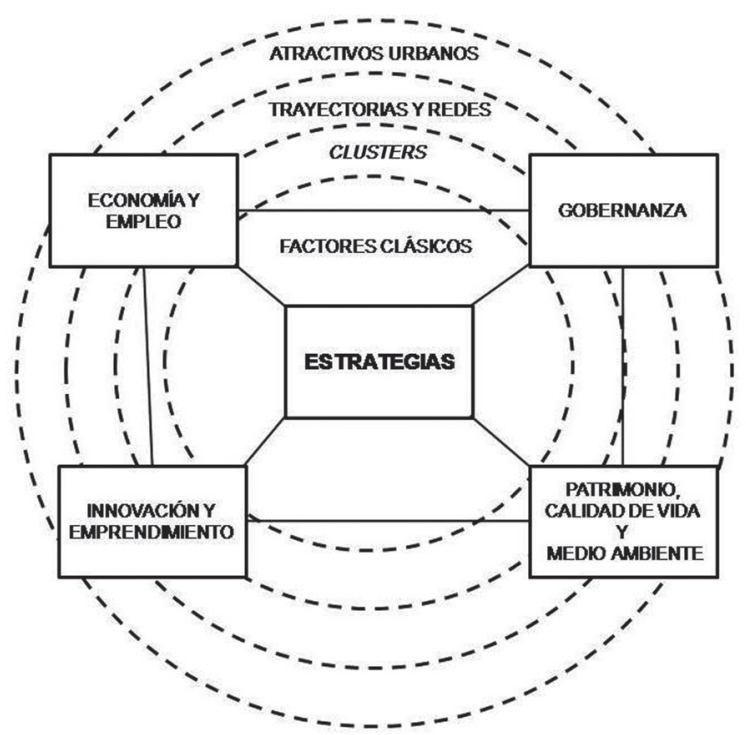

Fuente: Elaboración propia a partir de Méndez (2010) y

Musterd y Murie (2010).

otros) han conducido a cuestionar la validez de algunos de sus argumentos, especialmente de aquellos que hacen tabula rasa de las trayectorias y características territoriales y obviaban la larga y lenta transformación que requiere conseguir que este tipo de factores esté presente en las ciudades. Este hecho, no obstante, no impide que sea necesario valorar en su justa medida la presencia de estos factores en las ciudades (Musterd \& Murie, 2010: 28-29).

Esta interpretación del declive urbano y de las estrategias de desarrollo pone énfasis en la evolución concreta que sigue cada ciudad más allá de los procesos estructurales. En este sentido, resulta fundamental poner el valor de los casos de estudio tanto para interpretar las dinámicas de las ciudades desde el punto de vista que acaba de plantearse como para interpretar estas dinámicas desde investigaciones que vinculen el análisis de los fenómenos globales, a través de datos estadísticos con los estudios locales, donde los procesos económicos tienen lugar a través de sus manifestaciones específicas (Crang, 2002: 648), poniendo en valor el estudio de caso como medio para acceder a un conocimiento profundo y válido de la realidad (Baxter \& Eyles, 1997). Habi- tualmente, se presta una especial atención a las trayectorias locales en forma de estructuras económicas o sociales heredadas a través del concepto de path dependence propuesto por la Geografía Económica Evolutiva. Un proceso o sistema de path dependence ${ }^{5}$ es aquel cuya dinámica evoluciona como consecuencia de la propia trayectoria que sigue el sistema (Martin \& Sunley, 2006: 399). Es decir, las decisiones tomadas en el pasado influyen en la posibilidad de tomar nuevas decisiones, de forma que la dinámica de una ciudad no se considera una secuencia rígida, determinada por la tecnología o el pasado, sino una especie de «mapa de carreteras» en el que ciertas direcciones aparecen señaladas más fácilmente que otras (Walker, 2001: 126). Muy relacionado con esto se encuentra el concepto de lock-in ${ }^{6}$, que hace referencia a la persistencia de ineficiencias en los sistemas o economías como consecuencia de la trayectoria seguida. En este sentido, una evolución también puede suponer que determinadas disposiciones institucionales puedan permanecer bloqueadas

\footnotetext{
5 Que se traducirá como "trayectoria urbana".

6 Que se traducirá como "bloqueo interno" o "Iastre".
} 
por períodos de tiempo considerables a pesar de existir alternativas más eficientes (Grabher, 1993; Simmie \& Carpenter, 2008). Para el caso de las ciudades de tradición industrial en declive, las estructuras económicas heredadas del pasado o la existencia de actitudes reacias a cambiar el modelo productivo pueden repercutir tanto en la evolución de estos espacios como las decisiones de los actores locales, constituyendo bloqueos internos que influyen y condicionan las estrategias de revitalización.

De este modo, las condiciones locales y la dinámica interna de las ciudades pueden entenderse como elementos fundamentales para comprender la evolución de las estrategias de revitalización y también la propia vulnerabilidad y capacidad de reacción de una ciudad frente a un shock externo. En este sentido, puede recurrirse a la metáfora de resiliencia urbana, que se define como la capacidad de las ciudades para transformarse frente a ciertas sacudidazas que afectan negativamente a su base económica, produciéndose, en esencia, una reinvención de su economía (Polèse, 2010: 8). Así, queda de manifiesto la capacidad que muestran algunas ciudades para enfrentarse a procesos de declive y revertirlos, lo que supone un incremento de sus ventajas competitivas, pero también de su cohesión social interna, sus procesos de gestión local, su calidad de vida y su sostenibilidad, aumentando de ese modo las posibilidades de atraer población, inversiones y empresas que puedan generar un nuevo dinamismo en estos espacios (Méndez, 2012: 220). Esta idea se refiere a la desigual capacidad de los lugares para reaccionar, responder y hacer frente a incertidumbres de distinta naturaleza y que provocan profundos y rápidos cambios en la economía local (Pike et al., 2010: 59). Por ello, resulta muy útil a la hora de interpretar la desigual evolución de las ciudades en declive y su capacidad para enfrentarse a una nueva crisis económica como la actual. Así, los casos de Langreo y Avilés son ilustrativos de estas ideas y constituyen ejemplos válidos sobre la necesidad de una profunda reflexión en la definición de las estrategias locales y en las posibles líneas de acción en las iniciativas para revertir el declive urbano.

Para llegar a una correcta interpretación de las dinámicas locales de estas ciudades, no aprehensibles tan solo a través del uso de estadísticas, publicaciones, documentos de instituciones públicas y privadas, etc., se recurrió a las entrevistas a actores locales, quienes a menudo aportaron algunas claves que no se reflejaban en las estadísticas o no aparecían de manera suficientemente explícita en las obras científicas. En este sentido, un punto importante fue la entrevista a un «informante clave», una técnica estándar ampliamente usada en este tipo de estudios. Este término se aplica a cualquier persona que pueda brindar información detallada debido a su experiencia o conocimiento de un tema específico. Se considera que la entrevista con el informante clave al comienzo del estudio puede facilitar el tener una idea clara de los temas pertinentes, obteniéndose una imagen general aproximada de la ciudad a estudiar. Además, permite identificar a otras personas o instituciones relevantes para el tema de estudio, sugiriendo potenciales entrevistados en un proceso conocido como "de bola de nieve» (Biernacki \& Waldorf, 1981). En este sentido, se identificó y entrevistó a un informante clave en cada ciudad ${ }^{7}$, relacionado de manera amplia con la realidad de estos espacios y con la suficiente capacidad como para aportar una primera idea general que orientase el desarrollo de la investigación. A partir del mismo se construyó el trabajo de campo, en base a referencias (artículos, estudios, dossieres, etcétera.) localizadas desde diferentes ámbitos (revistas especializadas, obras locales o regionales, documentos en las webs institucionales, etcétera), que junto a la información de la prensa regional o local y de los recursos electrónicos consultados, permitieron estructurar este proceso de trabajo y las entrevistas a la muestra de actores seleccionada.

De esta manera, se diseñó una muestra de actores en base a algunos estudios previos (Alonso y Méndez, 2000; Prada, 2011) que proponen la utilización de un «diamante de actores» con el objetivo de cubrir de la manera más amplia posible el abanico de agentes que actúan sobre el territorio. Dicho

\footnotetext{
7 En el caso de Langreo, el informante clave fue el Dr. Aladino Fernández, Geógrafo de la Universidad de Oviedo y alcalde de Langreo entre 1983-1987, y en Avilés fue Dña. Pilar Varela, actual alcaldesa de la ciudad. El autor quiere expresarles su agradecimiento.
} 
diamante, adaptado a las características de cada caso de estudio, reúne a actores políticos, económicos y sociales, que fueron entrevistados para cubrir un amplio espectro de temáticas y perfiles. Los criterios para la identificación de los actores entrevistados fueron múltiples. Por un lado, se tuvieron en cuenta las indicaciones del informante clave entrevistado al comienzo del proceso, quien aportó diferentes nombres, instituciones, organizaciones, etc., de interés. Por otra parte, se fijaron algunas prioridades, relacionadas con la necesidad de entrevistar a representantes del gobierno local y de la oposición política, de los principales sindicatos, asociaciones vecinales o profesionales, medios de comunicación y centros de investigación, de empresas o vinculadas de otra manera con el desarrollo. De esta forma, se pretendía cubrir un abanico amplio en cuanto a tipologías de actores.

Para el análisis de estas redes y de los actores que las componen se concertaron entrevistas, enviándose cartas y, a partir del contacto telefónico y vía correo electrónico, se produjeron varios desplazamientos a Langreo y Avilés, habiéndose elaborado previamente unos guiones en base a toda la información disponible. Dichos guiones se realizaron de forma que contuviesen una base de preguntas similares y comparables entre sí, junto con otras que hicieran referencia al campo de especialización de cada actor, para poner el acento en diferentes problemáticas urbanas. Entre las primeras se encontraban epígrafes referentes a la participación en proyectos, la colaboración con otros actores locales y extralocales, la opinión sobre la evolución de la ciudad en los últimos años y las causas de dicha evolución o las estrategias y políticas destacadas, mientras que las segundas fueron más heterogéneas.

\section{Crecimiento, crisis y declive en dos ciudades de tradición minero-industrial: los casos de Langreo y Avilés}

Los dos casos de estudio analizados se encuentran en Asturias, una región española de tradición industrial que sufrió en los años setenta y ochenta del siglo XX un duro proceso de ajuste en su sector secundario.
El interés de Langreo y Avilés se debe a que ambas ciudades crecieron gracias al impulso de la industria y padecieron posteriormente una importante crisis con consecuencias negativas sobre su desarrollo. Esta crisis afectó a su evolución demográfica, su economía local, su crecimiento urbano y su medio ambiente en la segunda mitad del siglo XX. Sin embargo, las estrategias de revitalización seguidas por ambas ciudades desde entonces para enfrentar estos retos fueron diferentes. El resultado han sido unas trayectorias distintas con consecuencias visibles en la evolución de los indicadores demográficos, económicos y sociales.

La región de Asturias, en el norte de España, sufrió desde mediados del siglo XIX un temprano proceso de industrialización en relación al resto del país como consecuencia de la existencia de importantes yacimientos de carbón en su subsuelo. En la costa (Gijón) y en los valles del interior de Asturias (Langreo y Mieres) se desarrollaron explotaciones mineras e industrias siderúrgicas que atrajeron otras empresas auxiliares y nuevas inversiones. En unas décadas se configuró un sistema urbano donde el espacio central (dominado por la capital de servicios, Oviedo, y por los principales centros económicos) creció en detrimento de las áreas occidental y oriental de la región, que sufrieron un intenso proceso de despoblamiento (Figura $\mathrm{N}^{\circ} 2$ ).

La ciudad de Langreo, hasta entonces formada por pequeños núcleos dispersos en el fondo de un valle, creció hasta superar los 66.000 habitantes en 1960. Langreo se convirtió en el principal productor de carbón de España, atrayendo un fuerte contingente de población inmigrante. Sin embargo, como consecuencia del rápido e intenso proceso de industrialización y de la escasa disponibilidad de suelo, aparecieron problemas urbanísticos y ambientales muy graves que persisten aún hoy en la ciudad. Entre los más importantes se encuentran aquellos relacionados con la mala calidad de la vivienda y la escasez de equipamientos, la contaminación del río Nalón (que discurre en paralelo al valle), la presencia de explotaciones mineras, empresas siderúrgicas y una central térmica junto a espacios residenciales, o la proliferación de viviendas de autoconstrucción en las laderas del valle (Prada, 2011). 
Figura $\mathrm{N}^{\circ} 2$

Red urbana de Asturias

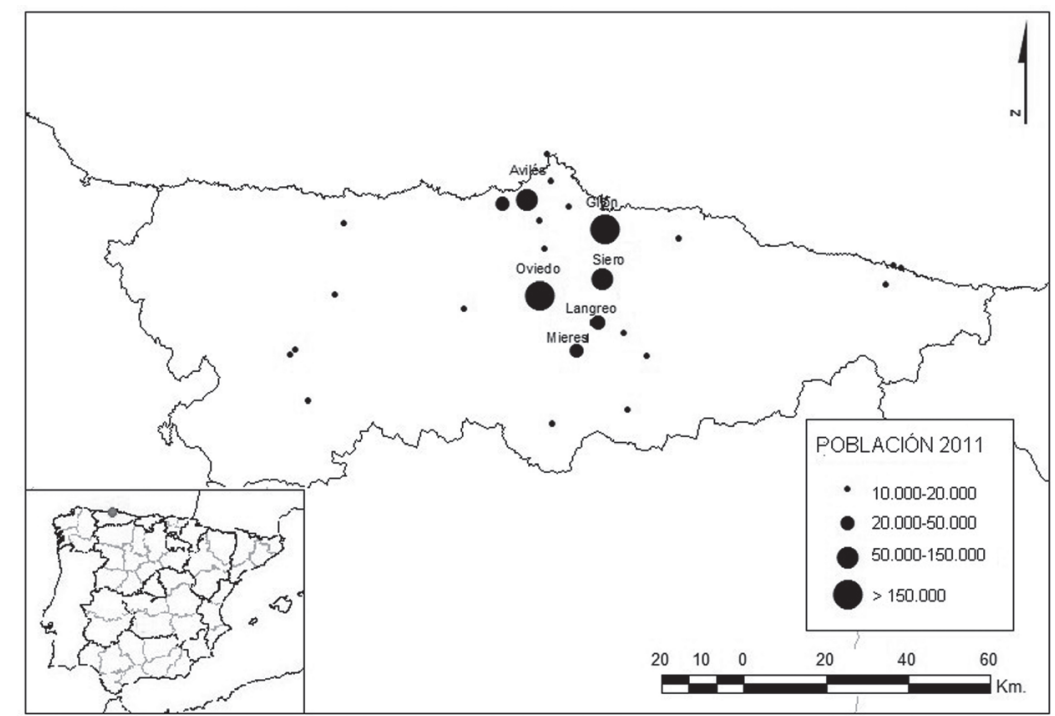

Fuente: Elaboración propia a partir de Padrón Municipal de Habitantes (2011).

Durante el siglo XX se produjo una intervención creciente del Estado en la economía asturiana a través del sector industrial. Como consecuencia, en la década de los 50 el Gobierno de España decidió la construcción de un complejo siderúrgico integral en Avilés. En ese momento la ciudad tenía una población de apenas 21.000 habitantes. Con el inicio de las obras pasó a convertirse en uno de los puntos de mayor inmigración del país hasta comienzos de la década de 1970, cuando el flujo inmigratorio se frenó. Entonces la población de Avilés superaba los 85.000 habitantes, lo que tuvo un impacto demográfico, social y cultural considerable. El legado de este proceso de industrialización (y del subsiguiente fenómeno de desindustrialización) fue profundo, siendo sus consecuencias visibles aún hoy. En primer lugar, la llegada de población inmigrante condujo a la creación de un conjunto de nuevos barrios y urbanizaciones en la ciudad. Su rápida construcción y su escasa planificación supusieron la aparición de déficits en cuanto a equipamientos, servicios, conexiones de agua y luz o deficiencias en las propias viviendas que persistieron durante años. Además, estos espacios presentaban una importante segregación social y funcional de los trabajadores en base a la distribución jerárquica de la compañía, de modo que las diferencias entre unas categorías sociales y otras eran considerables. Finalmente, las nuevas urbanizaciones contaban con sus propios hospitales, escuelas, economatos y servicios, de uso exclusivo para los empleados, de manera que no se dio una integración entre la población autóctona y los recién llegados, tratados habitualmente con cierto menosprecio por estos (Sánchez et al., 2012). Avilés sufrió también una intensa degradación de su calidad ambiental, especialmente en su ría, convertida en el eje sobre el que se desarrolló la actividad siderúrgica, de forma que pasó a ser una de las ciudades más contaminadas de España.

Sin embargo, en la segunda mitad del siglo XX ambas ciudades se enfrentaron a un proceso de desindustrialización y a una crisis económica, demográfica y social. En primer lugar, Langreo se vio afectado por la apertura económica de España a partir de 1960. Por un lado, se produjo el colapso de las empresas mineras y el Estado decidió en 1967 su compra y agrupación en un gran consorcio público, Hulleras del Norte Sociedad Anónima (HUNOSA). A partir de ese momento dio inicio a unos Planes de Ajuste en el sector, destinados a reducir la población ocupada en la minería y la producción de carbón. El 
valle del Nalón pasó así de ocupar a más de 25.000 mineros en la década de los 50 a unos 1.000 en la actualidad.

Por otro lado, la industria siderúrgica de Langreo, incapaz de competir en un contexto de apertura económica, sufrió un proceso similar al de la minería. Tras la nacionalización progresiva del sector en torno a la empresa pública Empresa Nacional Siderúrgica Socidad Anónima (ENSIDESA), las nuevas inversiones se dirigieron hacia la costa (a Avilés y Gijón). Con esto se buscaba crear nuevas acerías más eficientes, de mayor tamaño, y vinculadas al transporte marítimo frente al modelo siderúrgico "a pie de mina» existente en Langreo y Mieres. Las fábricas de Langreo se desmantelaron progresivamente hasta su cierre definitivo en 1983. Estos cierres afectaron también a aquellas actividades dependientes de la minería y la siderurgia. Así, las empresas químicas y las pequeñas y medianas empresas dedicadas a los transformados metálicos comenzaron a cerrar debido al efecto que sobre ellas ejerció el desmantelamiento de las grandes compañías estatales. Por último, el escaso comercio existente en Langreo (en 1960 el conjunto del sector servicios empleaba tan solo al $14,7 \%$ de la población activa) se vio afectado por la pérdida de empleos y la masiva emigración de trabajadores y jóvenes.

Por su parte, Avilés se vio sacudida por la crisis económica de los años setenta. Al igual que otras ciudades y regiones europeas de antigua especialización, Avilés tuvo que hacer frente a problemas de competitividad de la industria que, hasta entonces, había sustentado su crecimiento. Dicha industria tuvo que adaptarse a un nuevo contexto productivo marcado por nuevas formas de organización y por cambios en las estrategias de competitividad de las empresas, incluyendo innovaciones tecnológicas más acusadas, segmentación de los procesos productivos y deslocalización de determinadas ramas de su actividad. Con retraso respecto de otras acciones emprendidas en Europea, las autoridades españolas acometerían un primer Plan de reconversión de la siderúrgica integral (1984-1990), centrado en las empresas ENSIDESA y Altos Hornos de Vizcaya, o lo que es lo mismo, Avilés y Bilbao, este segundo caso convertido en ejemplo paradigmático de la reconversión industrial y de las estrategias de revitalización en España (González, 2006) y referencia para la actual estrategia de desarrollo urbano de Avilés analizada en este trabajo. En una segunda fase (1991-1997) se produjo la desaparición de ENSIDESA en 1995 que, al igual que el resto de siderurgias españolas, quedaba englobada en la Corporación Siderúrgica Integral (CSI), participada al $50 \%$ por el capital público. Tras un proceso de saneamiento y redimensionamiento, la empresa fue vendida en 1997 a Aceralia, de capital luxemburgués, que en 2002 se integraba en la multinacional Arcelor-Mittal. Como consecuencia, el número de trabajadores empleados en la industria descendió bruscamente, provocando el aumento del paro. Los ajustes realizados por ENSIDESA en Avilés hicieron que esta pasase de 15.200 empleos en 1990 hasta unos 3.000 en la actualidad.

La consecuencia fue una ruptura con las trayectorias de crecimiento continuo que Langreo y Avilés habían experimentado durante el siglo XX. En este contexto, la población de Langreo en 1960 era de 66.323 habitantes, disminuyendo hasta los 44.737 en 2011, lo que significa un descenso de más del $30 \%$ en los últimos 40 años (Figura N³). En paralelo, el declive demográfico ha supuesto un brutal envejecimiento de la población, de forma que actualmente más de un $25 \%$ de la población langreana supera los 65 años frente al escaso $11 \%$ cuya edad se encuentra por debajo de los 16 años.

En el caso de Avilés, la sucesión de dos décadas de descenso de su población supone una reducción del 4\% de su población entre 1981 y 2001, alrededor de 3.400 habitantes (Figura $N^{\circ} 3$ ). En la última década, sin embargo, la ciudad ha vuelto a registrar un modesto crecimiento. Este, que se debe a un flujo migratorio positivo que viene a compensar el decrecimiento natural de su población, ha supuesto un crecimiento del $0,5 \%$ en su población total entre 2001 y 2011. A pesar de que este dinamismo es mucho menor que el de Oviedo y Gijón (más diversificadas desde un punto de vista funcional), representa una ruptura con su trayectoria reciente, indicando unos cambios sustanciales durante los últimos años y una evolución mejor que la de Langreo, que ha perdido un 2,1\% de su 
Figura $\mathrm{N}^{\circ} 3$

Evolución demográfica en Langreo y Avilés (1877-2011)

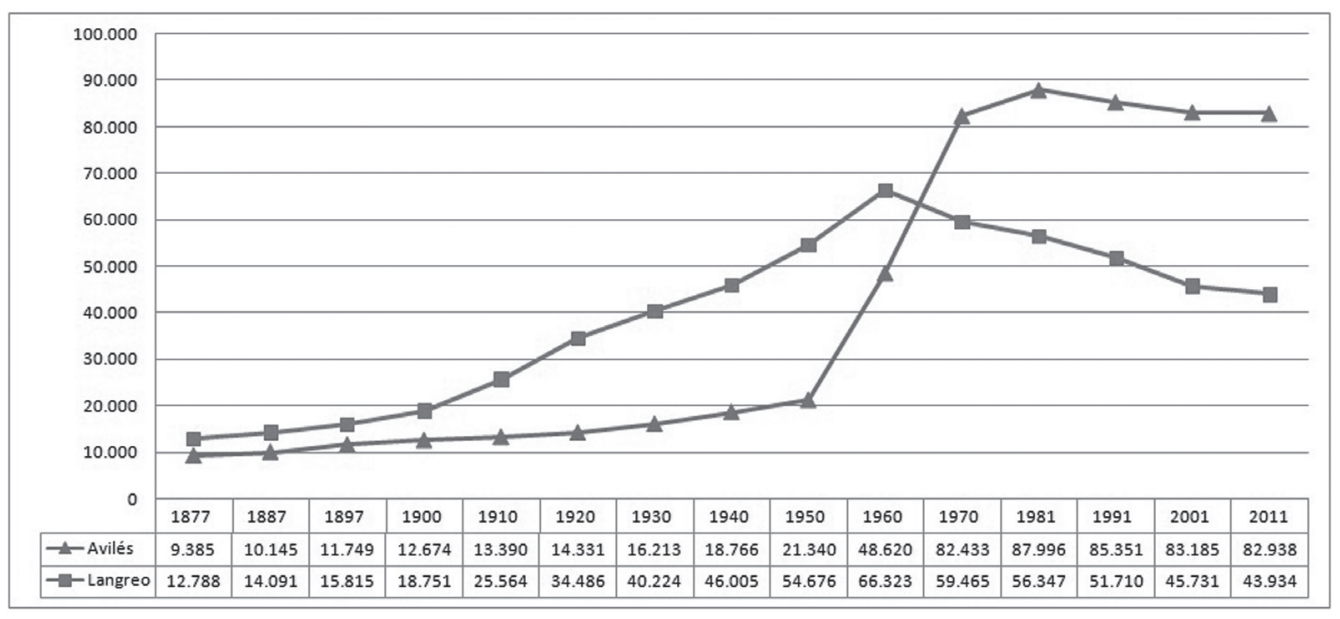

Fuente: Elaboración propia a partir de Censos de Población y Padrón Municipal de Habitantes (varios años).

población en esta década. Sin embargo, el envejecimiento de la población de Avilés es también acusado, encontrándose en la actualidad el $21 \%$ de la población por encima de los 65 años frente al 12\% con menos de 16 años.

Si la dinámica demográfica ha sido desigual, algo similar ha ocurrido con la evolución del empleo en ambas ciudades (Cuadro $N^{\circ} 1$ ). Los datos disponibles referidos a la dinámica del empleo total muestran cómo, a pesar de que entre 1990-1995 tanto Avilés (-20\%) como Langreo (-24\%) pierden empleo, el impulso de la recuperación a partir de entonces es diferente. Mientras que Langreo incrementa el número de empleos un 6,9\% entre 1990-2005 (momento previo a la crisis de 2008), Avilés se muestra mucho más dinámica $(18,6 \%)$. El resultado es una mejor evolución de Avilés, a pesar de que los datos de ambas ciudades de 2010 muestran un cambio de trayectoria como consecuencia de la mencionada crisis económica.

Un indicador final sobre la profundidad del proceso de revitalización en Langreo y Avilés puede ser la evolución del precio de la vivienda en estas dos ciudades. En 1994 el precio de la vivienda en Avilés y Langreo era muy similar (tan solo una diferencia de 23 euros por $\mathrm{m}^{2}$, entre ambas ciudades). Sin embargo, en 2011, el precio en Avilés se había incrementado hasta cifras similares a las de las principales ciudades regionales, Oviedo y Gijón, mientras que el de Langreo lo había hecho más modestamente, con lo que la diferencia pasaba a ser de 158 euros por metro cuadrado. Además, tras el proceso de crisis y explosión de la "burbuja inmobiliaria" española, la caída del precio de la vivienda en Avilés ha sido mucho menor que en Oviedo y Gijón, lo que en conjunto confirma la mejora de Avilés como un lugar atractivo para vivir.

En ambas ciudades existen otras manifestaciones de los procesos de revitalización puestos en marcha. La importante actuación pública en aspectos como la mejora de los servicios y equipamientos, la economía y el empleo, o el urbanismo y la cohesión social deben ser mencionadas aquí. Estas dimensiones, fundamentales para comprender los procesos de revitalización acaecidos en Langreo y Avilés se desarrollan en los puntos siguientes. A partir de las mismas se llevará a cabo una interpretación de los elementos clave en estos procesos, identificando a los actores que se han destacado en la construcción de redes locales de colaboración o en la promo- 
Cuadro $N^{0} 1$

Evolución del empleo en Avilés y Langreo (1990-2010)

\begin{tabular}{|l|c|c|c|c|r|r|r|r|}
\hline & 1990 & 1995 & 2000 & 2005 & 2010 & $\begin{array}{c}\text { Variación (\%) } \\
1990-1995\end{array}$ & $\begin{array}{c}\text { Variación (\%) } \\
1995-2005\end{array}$ & $\begin{array}{c}\text { Variación (\%) } \\
1990-2010\end{array}$ \\
\cline { 1 - 7 } Avilés & 32.971 & 26.411 & 28.868 & 31.324 & 29.279 & $-19,9$ & 18,6 & $-11,2$ \\
\cline { 1 - 5 } Langreo & 15.844 & 12.011 & 12.242 & 12.838 & $12.999 *$ & $-24,2$ & 6,9 & -18 \\
\hline
\end{tabular}

*Aunque el dato de 2010 es superior al de 2005, representa un descenso respecto a 2006, 2007, 2008 y 2009. Fuente: Elaboración propia a partir de datos de SADEI.

ción exterior de la ciudad y poniéndolas en relación con el punto anterior.

\section{Luces y sombras en los procesos de revitalización: el peso de las actuaciones locales}

Durante varias décadas Langreo y Avilés han estado inmersos en un proceso de revitalización económica y regeneración urbana para revertir las dinámicas negativas que originaba el declive urbano. Aunque cualquiera de estos procesos se ve influenciado por múltiples factores, lo que se subraya en este artículo son el papel y las estrategias de los actores locales, en base al esquema desarrollado en el marco teórico (Figura $\mathrm{N}^{\circ}$ 1). Sin embargo, también se hará referencia a algunas actuaciones destacadas en otras dimensiones. Una de las características que comparten los dos casos de estudio ha sido el aumento del número de actores y de su implicación en las estrategias de desarrollo. Actores externos y, sobre todo, locales tienen hoy un importante peso explicativo en las políticas adoptadas y en la trayectoria de Langreo y Avilés, tal y como otros estudios de referencia han puesto de manifiesto (Pinson, 2009: 349-352). A partir del trabajo de campo y de los resultados de las entrevistas a actores locales, se desarrollan e interpretan las estrategias de revitalización llevadas a cabo en ambas ciudades.

En primer lugar, en aquellas estrategias que tienen como objetivo el desarrollo económico y la creación de empleo, la presencia o potenciación de factores clásicos es aún muy importante, como señalan para otros ca- sos Musterd y Murie (2010: 347). En Langreo ha tenido una gran relevancia la creación de nuevo suelo industrial y empresarial para tratar de mantener el empleo ligado al sector secundario. Este modelo, defendido principalmente por el Ayuntamiento y los sindicatos (con un peso importante en la comarca) ha tenido un impacto físico destacado. Como consecuencia de la escasa separación de usos y del poco suelo disponible en el fondo del valle se ha mantenido la convivencia entre espacios industriales y residenciales, creando un modelo de ciudad poco atractivo. Por otro lado, se ha producido una mejora de las comunicaciones hacia el centro de Asturias, lo que ha generado la llegada de nuevas empresas a Langreo. Sin embargo, el escaso atractivo de la ciudad no ha favorecido el asentamiento de nuevos residentes, a pesar de la cercanía a los grandes núcleos asturianos y del precio más barato de la vivienda en Langreo. Finalmente, mediante las ayudas con fondos estatales a empresas para su instalación o ampliación se han reforzado o creado nuevos clusters económicos en la ciudad. Aunque se han realizado inversiones importantes en el sector de los transformados metálicos, que mantiene una larga trayectoria en Langreo, también se han desarrollado nuevos empleos relacionados con la atención sanitaria en base a las demandas de una población crecientemente envejecida. En síntesis, aunque la promoción económica y del empleo ha sido una prioridad para la ciudad, la mala planificación y la falta de espacios libres han tenido consecuencias negativas sobre otros aspectos que se analizarán a continuación.

En Avilés se ha apostado también por las inversiones en «factores clásicos» para la 
creación de empleo. En este sentido, destaca la construcción del Parque Empresarial del Principado de Asturias sobre antiguos terrenos de la industria siderúrgica, impulsada por el Estado con apoyo del gobierno regional. Esta actuación ha sido muy importante para la llegada de nuevas empresas a la ciudad, a pesar de que algunos entrevistados señalaron déficits relacionados con la gestión del suelo o la ubicación de las empresas. Otro elemento fundamental en la revitalización económica de Avilés ha sido la ampliación y diversificación funcional de su puerto, que ha contado con el consenso de todos los actores. Para explotar todo el potencial del puerto, los planes de futuro se centran en la ampliación del mismo hacia la margen derecha. De esta forma, mientras que el área más alejada de la ciudad pasaría a acoger las actividades más relacionadas con la industria, los terrenos libres de la margen izquierda se dedicarían a otros usos con menor impacto sobre la ciudad (lonja, puerto deportivo, área de servicios, etcétera) en un fenómeno que guarda paralelismos con el proceso de expulsión de actividades obsoletas y su reemplazo por servicios y actividades económicas más innovadoras en Barcelona durante las últimas décadas. Finalmente, entre las estrategias más importantes desarrolladas por los gobiernos regional y local se encuentra la promoción turística de Avilés. Un cluster económico en torno a este sector se ha consolidado mediante la recuperación de recursos existentes e infrautilizados turísticamente (casco histórico, ría, fiestas tradicionales) y la creación ex novo de otros (destaca el Centro Cultural Internacional Oscar Niemeyer, que se comenta más adelante). De esta forma, algunas de las estrategias puestas de manifiesto en la literatura científica al uso (Méndez, 2010; Musterd \& Murie, 2010) han tenido su reflejo en las dos ciudades, adaptándose a unas realidades que, en el caso de Langreo, han quedado tamizadas por la existencia de fuertes intereses locales para el mantenimiento de una potente base industrial, como se verá después.

En segundo lugar, en ambas ciudades pueden encontrarse medidas destinadas a fortalecer la innovación y la emprendeduría, objetivos fundamentales en las estrategias de desarrollo urbano. Estas cuestiones son esenciales para la competitividad urbana, pero también tienen un potencial clave para el cambio tecnológico y la evolución industrial (Cooke \& Simmie, 2005: 97-100). En el caso de las ciudades de tradición industrial, la obsolescencia económica y la falta de emprendedores son lock-ins o lastres que han afectado especialmente a su desarrollo (Martínez-Fernández et al., 2012), por lo que estas iniciativas son más importantes, si cabe. En este sentido, en Langreo destaca la actividad realizada por el Centro de Empresas de Valnalón, construido sobre los antiguos terrenos de la acería de la ciudad. Este centro, impulsado con el apoyo de actores locales y regionales (Ayuntamiento, sindicatos, gobierno regional) Ileva a cabo un amplio proyecto de formación de emprendedores mediante cursos desde el colegio para que los niños se familiaricen con la cultura emprendedora. Entre las instalaciones del centro se encuentran también un semillero de proyectos, una incubadora de empresas, naves y oficinas para los nuevos proyectos y aulas donde se imparte formación continua. El propio director, entrevistado durante el trabajo de campo, explicaba que la labor del centro es subsanar la carencia de emprendedores del Valle como consecuencia de una trayectoria marcada por más de un siglo bajo el "paraguas» de la gran empresa, lo que puede considerarse un lastre para el desarrollo de la ciudad:

"Hay muchos emprendedores sociales, pero falta el emprendedor económico. [En el Valle] tienes muchas asociaciones de todo tipo y mucho intraemprendedor, gente que está dentro de la empresa y da lugar a pequeñas innovaciones, pero las grandes empresas públicas inhibieron la aparición de emprendedores económicos".

En el caso de Avilés, la actuación de mayor calado con la intención de superar algunos bloqueos heredados de su trayectoria urbana en el fomento de la innovación y la emprendeduría es la puesta en funcionamiento del Instituto Tecnológico de Materiales, encargado de desarrollar proyectos de $\mathrm{I}+\mathrm{D}+\mathrm{i}$ conjuntamente con las empresas del sector del acero y de ofrecer servicios de formación especializada y de pruebas en laboratorio. Según su director, con ello las empresas encuentran ventajas a la hora de competir a escala internacional y de ubicarse en nichos de mercado especializados. Ambas ciudades tienen otras iniciativas en estos ámbitos, bien 
centradas en la superación de determinadas trayectorias donde la cultura emprendedora está poco desarrollada (proyecto de una Escuela de mujeres emprendedoras en Avilés) o bien favoreciendo la colaboración entre empresas e instituciones en proyectos de investigación. De esta manera, la política innovadora de ambas ciudades se ha dirigido a solventar debilidades arrastradas durante décadas (consecuencia de su trayectoria particular) y a reforzar sectores emergentes.

En tercer lugar, las medidas relacionadas con la calidad de vida, el urbanismo, el patrimonio minero-industrial y el medio ambiente resultan fundamentales en las ciudades de tradición industrial. Las consecuencias del proceso de industrialización (urbanismo caótico, escasez de equipamientos, contaminación o aparición de ruinas industriales) son una "pesada herencia» para ellas y un reto para iniciar un cambio de imagen y un nuevo dinamismo en espacios con una tradición industrial (Guasch y Zulaika, 2007: 5-20). En el caso de Langreo, como se dijo anteriormente, estos problemas no se han tenido en cuenta en la misma medida que otros aspectos a la hora de planificar la revitalización de la ciudad. Por un lado, pueden encontrarse actuaciones interesantes, destinadas a mejorar su aspecto, como el desarrollo de unos "Planes de fachadas» que se han llevado a cabo con fondos especiales del Estado, o la puesta en marcha de nuevos equipamientos de servicios, muchos de ellos realizados en una arquitectura de gran calidad, a través de fondos regionales, nacionales o europeos. La protección y reutilización del patrimonio minero-industrial ha sido otra pieza importante de la recuperación urbanística de Langreo. En primer lugar, se realizó un inventariado, catalogación y protección de los elementos existentes. A partir de este se ha planificado una serie de equipamientos (Museo de la Siderurgia, Pinacoteca Local, Centro de Formación Profesional, etcétera) o de actuaciones de protección sobre elementos singulares del patrimonio (bocaminas o castilletes mineros). El resultado ha sido una valorización del mismo y su conservación, algo que no ha sucedido en otras ciudades. Sin embargo, en paralelo se han obviado otros elementos que constituyen los principales problemas actuales de la ciudad en cuanto a su imagen y a la movilidad urbana. En primer lugar, se ha retrasado indefinidamente el soterramiento de las vías de tren que atraviesan la ciudad dificultando la movilidad de sus ciudadanos. A pesar de que las obras se iniciaron en 2009, la crisis económica ha reducido enormemente su presupuesto, de manera que esta actuación ha quedado paralizada. En segundo lugar, destaca la proliferación de nuevas áreas empresariales, impulsadas por Ayuntamiento, asociaciones de empresarios y sindicatos, en los entornos urbanos. En muchos casos se trata de antiguos espacios minero-industriales que fueron desmantelados en los años setenta y ochenta y que han conservado su uso a pesar de la proximidad a equipamientos o áreas residenciales. En tercer lugar, se encuentra la permisividad del Ayuntamiento local a la presencia de una central energética en el centro de la ciudad. Esta central, que parte en dos la trama urbana de Langreo, tiene su origen en la necesidad de energía para la fabricación de acero en los años cincuenta. A pesar de las protestas de ecologistas, asociaciones de vecinos o grupos políticos de la oposición, manifestadas en las entrevistas, el interés del Ayuntamiento local y de los sindicatos en mantener los puestos de trabajos de la central -50 en total- han evitado su desmantelamiento. En la actualidad está prevista su ampliación.

Por el contrario, las iniciativas destinadas a mejorar la calidad de vida, el medio ambiente y el urbanismo han jugado un papel crucial a la hora de mejorar la imagen de Avilés, ciudad que se ha inspirado en el modelo de Bilbao para poner en marcha una estrategia de desarrollo basada en la tecnología, el turismo y en una imagen innovadora. En primer lugar, destacan intervenciones más «físicas» como el Plan de recuperación del centro histórico, la renovación de edificios públicos (palacios, sedes municipales, mercados, etcétera) y la limpieza de la ría. El Plan de recuperación del centro de Avilés se ha llevado a cabo a lo largo de veinte años con fondos europeos, nacionales, regionales y locales, habiendo supuesto en conjunto una inversión de 21 millones de euros. Dentro de este plan se encuentran iniciativas encaminadas a renovar las viviendas y transformar edificios de gran valor en desuso en nuevos equipamientos o en instalaciones del Ayuntamiento de Avilés. Los trabajos de limpieza de la ría de Avilés comenzaron en 2003, y 
han requerido una inversión de más de 14 millones de euros para remover las toneladas de lodos contaminados depositados por ENSIDESA durante décadas. Estas actuaciones mediante intervenciones «duras» han sido importantes para subsanar carencias que se habían formado en la ciudad.

Destacan también otras políticas de barrio para atajar problemas heredados de la etapa de crecimiento desordenado y convertir a Avilés en una ciudad atractiva para vivir. Sobresale la intensa labor del Ayuntamiento local entre estas iniciativas, para la coordinación de acciones en distintas áreas como Salud, Infancia, Igualdad o Juventud, dentro de un Plan Rector de políticas para la cohesión social del municipio (desarrollado con instituciones sociales comarcales), que tiene en las políticas activas de vivienda un pilar esencial y que daría continuidad a la labor internacionalmente reconocida del Programa Municipal de Erradicación del Chabolismo, Realojamiento e Integración Social de la Población, iniciado a finales de los ochenta. De hecho, Avilés fue premiada en la calificación del BEST del VI Concurso de Naciones Unidas sobre Buenas Prácticas para Mejorar las Condiciones de Vida (2006). La existencia de una «trayectoria», como definió el concejal de Cultura de la ciudad a la labor realizada en la ciudad durante los últimos años, sería una de las claves de estas estrategias:

"Avilés ha cambiado casi sin darse cuenta, 'poco a poco' [...] Avilés tiene un grado de equipamientos (culturales, sociales, sanitarios y deportivos) difícil de igualar en cualquier otra ciudad de su tamaño y el reto en los próximos años será mantenerlos".

Por lo que respecta a la cultura, del conjunto de medidas emprendidas destaca, muy por encima del resto, el emblemático proyecto del Centro Cultural Internacional Oscar Niemeyer, cedido al Principado de Asturias por el arquitecto brasileño Oscar Niemeyer en agradecimiento por la concesión del Premio Príncipe de Asturias de las Artes en 1989 e inaugurado en 2011. En todo caso, este no sería el único ejemplo de promoción de infraestructuras específicamente culturales, pudiendo mencionar la creación de centros de arte, un museo de la ciudad, una escuela de música o un conservatorio. La actual estrategia de city-marketing estaría aprovechando el empuje que ha supuesto ese primer proyecto para situar a Avilés en el «mapa» de ciudades con proyección internacional (Castells y Borja, 2000), atrayendo personalidades famosas a la ciudad para ayudar a la promoción de otros aspectos presentes históricamente en Avilés, como la cultura, la música, las artes escénicas, el turismo o la gastronomía. Multitud de iniciativas dan cuenta del esfuerzo realizado en la última década, permitiendo la consolidación de un producto turístico en torno a la cultura ${ }^{8}$, como el Plan de Turismo Gastronómico, la recuperación del Teatro Palacio Valdés o el Certamen Nacional de Cortos. De forma complementaria, la ciudad habría adecuado en estos años su infraestructura turística.

Sin embargo, pueden encontrarse también determinadas cuestiones sin resolver, aunque su impacto es, en conjunto, menor que en el caso de Langreo. En primer lugar, existe una importante barrera ferroviaria entre el centro histórico y las nuevas áreas de expansión urbanística. Aunque se ha tratado de subsanar mediante varias pasarelas elevadas, aún no se ha llegado a un acuerdo con el Ministerio de Fomento para su eliminación. En segundo lugar, en Avilés el patrimonio industrial no ha tenido la misma atención que en el caso de Langreo, habiéndose incluso destruido determinados vestigios del período industrial. Finalmente, la presencia de las baterías de cok, alquiladas por la empresa Arcelor-Mittal a la Sociedad Estatal de Participaciones Industriales (SEPI) hasta 2017, con una posible extensión hasta 2020, es objeto de controversia. A pesar de que inicialmente estaba planteada la desaparición de estas baterías en 1997, el fuerte incremento sufrido por el cok, la es-

\footnotetext{
8 Una idea que de forma recurrente aparece en las entrevistas alude a la existencia de un sustrato cultural importante en el pasado de la ciudad, lo que en tiempos le valió a Avilés el apelativo de la «Atenas del Norte». Su origen estaría en las inversiones realizadas por los emigrantes retornados («indianos»), sobre todo a finales del siglo XIX, para mejorar la vida cultural de la ciudad. Para algunos durante los años de la dictadura franquista se trató de borrar este legado cultural, al cual parece que la ciudad apela de nuevo, lo que constituye otra de las «trayectorias locales» detectadas durante el trabajo de campo.
} 
casez de otras baterías similares en Europa y las dificultades para construir nuevas debido a los altos estándares medioambientales que promueve la Unión Europea, hicieron muy rentable el mantenimiento de este complejo en Avilés. En consecuencia, diferentes voces críticas se han alzado en ocasiones en contra del impacto sobre la atmósfera y el paisaje de la comarca, especialmente debido a la proximidad de las mismas respecto del Centro Internacional Oscar Niemeyer.

En síntesis, en ambas ciudades existen redes y trayectorias de colaboración entre actores más o menos explícitas tras las actuaciones más «tangibles», tanto en las estrategias de mejora de la calidad de vida urbana, como en el desarrollo económico y en el fomento de la innovación. Estas se sustentan en una «cultura de la gobernanza» que, si bien no es siempre inherente a los territorios, puede fomentarse mediante distintas iniciativas con una proyección temporal (Kooiman, 2003).

En este sentido, las estrategias Ilevadas a cabo en Langreo con el objetivo de impulsar la gobernanza han tenido problemas para mantenerse a lo largo del tiempo. Las iniciativas más importantes se han desarrollado a nivel comarcal. El fuerte crecimiento durante la etapa de industrialización, el carácter lineal del valle y el escaso suelo disponible dieron lugar a la unión física de los núcleos urbanos del valle y a una estrecha relación entre ellos. En consecuencia, durante los años ochenta se impulsó una «Mancomunidad del Nalón» que reunía a los alcaldes de los cinco municipios con la intención de avanzar progresivamente hacia un Ayuntamiento único. En sus primeros años algunos servicios pasaron a desarrollarse de forma conjunta, sin embargo, la Mancomunidad del Nalón Ileva muchos años estancada por falta de voluntad política. La mayoría de los actores entrevistados han señalado la necesidad de avanzar hacia el Ayuntamiento único para reducir las duplicidades, ordenar de forma unitaria el territorio o lograr mayor peso político y recursos a nivel regional. No obstante, también han admitido la escasa voluntad de los dirigentes locales de ceder poder en favor de un Ayuntamiento único para el valle:

"La Mancomunidad pudo ser una buena herramienta para la cohesión de la comar- ca y avanzar hacia el Ayuntamiento único [...] Fue la primera de Asturias [...] Los resultados fueron muy pocos por intereses políticos muy grandes y falta de voluntad del Partido Socialista a nivel local y regional para desarrollar este ejemplo [...] En los Ayuntamientos hay mucho voto cautivo con el empleo en la administración local [...] Si se reducen los Ayuntamientos todo ese voto se pierde".

Por otro lado, en la década de los noventa se creó un Consejo de Desarrollo Comarcal con el objetivo de establecer líneas comunes de trabajo entre los agentes sociales para el desarrollo del Valle. Estuvo formado por sindicatos, representantes de los cinco ayuntamientos del Valle, del Principado de Asturias, representantes de los principales partidos políticos, empresarios, comerciantes e investigadores de la Universidad de Oviedo. Sin embargo, a pesar del interés de este organismo, hace años que dejó de convocarse y hoy no tiene ninguna presencia, habiendo pedido los sindicatos en varias ocasiones su recuperación. El ejemplo de Langreo resulta, de este modo, paradigmático en cuanto a anquilosamiento de unas estructuras sociales y políticas que hace unas décadas eran muy dinámicas, pero que se han ido cerrando con el paso del tiempo ante las limitaciones para incorporar mecanismos de seguimiento y control o de entrada de nuevos actores. Por el contrario, en Avilés se ha dado un proceso de toma de progresiva toma de importancia por parte de nuevos actores, en un proceso detectado en otras ciudades como Nantes (Pinson, 2009: 264-265). Un ejemplo de esto es la implicación creciente del puerto de Avilés en varias iniciativas (saneamiento de la ría, ampliación del propio puerto, Centro Cultural Oscar Niemeyer...) y su colaboración con distintos actores (Ayuntamiento de Avilés, Fundación del Centro Niemeyer). De esta forma, el entramado de actores de la ciudad se ha diversificado y ha dado entrada a nuevos integrantes. Como señalaba un entrevistado:

"Este no es el país de las maravillas, hay roces, piquillas, etc. pero como en todos lados, creo que intentamos siempre superarlos y desde esta institución lo hacemos [...] En cuanto al liderazgo político y social, este fue asumido por el Ayuntamien- 
to, entre otras cosas porque es el único que podía asumirlo".

No obstante, varios entrevistados han lamentado la inexistencia de órganos estables de concertación entre los actores, así como de una mayor «cultura de colaboración» a nivel comarcal. En este sentido, existe una iniciativa muy exitosa de concertación comarcal en el ámbito del turismo: la creación de la Mancomunidad Turística de Avilés y Comarca. Esta, a partir de la unión de cuatro ayuntamientos colindantes ofrece de manera unitaria todas las ofertas turísticas de la comarca y constituye un ejemplo pionero de concertación de servicios. A pesar de ello, varios entrevistados lamentaron que no existiesen iniciativas similares en otros aspectos clave para el desarrollo territorial de la comarca como la planificación del suelo o la gestión de las infraestructuras de transporte.

Sin embargo, en ambos casos, más allá de estas iniciativas concretas, el trabajo de campo ha mostrado la desigual trayectoria de los actores locales en cuanto a la concertación del modelo de desarrollo de cada caso. En Langreo, como ha podido verse en este punto, no ha existido un consenso entre los actores locales para concertar el modelo de ciudad, y los sindicatos y el Ayuntamiento, junto con otros actores, han promovido un gran número de iniciativas para crear empleo en detrimento de una mejora de la calidad de vida urbana. Esto ha provocado las críticas de varias asociaciones de vecinos, culturales y ecologistas, que se han opuesto a determinados proyectos sin lograr llegar a un acuerdo con el Ayuntamiento. De esta manera, se ha perdido la oportunidad de consensuar un modelo de desarrollo alternativo para la ciudad, elemento fundamental en las estrategias de innovación y competitividad urbana (Musterd \& Murie, 2010: 344-349). Como consecuencia, Langreo está considerada hoy una ciudad poco atractiva para vivir. Varios entrevistados admitieron que la ciudad tenía dificultades para atraer o mantener población a pesar de la mejora en las comunicaciones, de las actuaciones urbanísticas, del precio más bajo de la vivienda, de la creación de empleo en los últimos años o de la mejora en los servicios. También hacían referencia a la escasa capacidad para concertar un modelo de desarrollo alternativo con el Ayuntamiento, y las consecuencias negativas de esto sobre el desarrollo y el «ambiente» de la ciudad:

"Hay un déficit en la ordenación del territorio que viene de los criterios precarios con que se construyó la ciudad [...] la reindustrialización no vino a [...] romper con esto porque lo sindicatos lo han dirigido así y no ha tenido componentes ambientales [...] dejó lo económicamente rentable [...] la industria siguió ubicada en sitios pésimos para la población".

Por el contrario, uno de los grandes logros que ha conseguido Avilés ha sido la concertación de un modelo alternativo al de las tesis reindustrializadoras, con gran influencia en la ciudad hasta mediados de la década de los noventa. Encabezados por el Ayuntamiento, los principales actores han sido capaces de llegar a acuerdos básicos sobre la necesidad de desarrollar nuevos sectores (turismo) en paralelo al mantenimiento de las industrias más competitivas o sobre la importancia de invertir en equipamientos y la mejora del aspecto de la ciudad junto a los nuevos desarrollos de suelo empresarial. En consecuencia, la concertación de un modelo de ciudad entre los actores no solo ha permitido que esta se haya desarrollado de forma más consensuada. Avilés ha comenzado a atraer a residentes y visitantes y ha logrado un mejor «clima» en el desarrollo de una gobernanza urbana, aspecto que ha sido incluso reconocido internacionalmente ${ }^{9}$. Esto no ha evitado que hayan surgido algunas discrepancias, en especial en relación a la pervivencia de las baterías de cok, ya comentada, pero la mayoría de los entrevistados ha coincidido en señalar el importante papel del Ayuntamiento para llegar a acuerdos y mediar entre actores:

"Durante veinte años hemos estado dando bandazos para un sitio y para otro sin saber lo que queríamos. Que ahora la gente, sobre todo los sindicatos (que eran los más reivindicativos y los que salieron a la calle) digan "ahora sabemos lo que queremos" es importante [...] En Asturias no hay nada que se pueda comparar a Avilés".

\footnotetext{
9 En 2007 Avilés fue reconocido como ejemplo de buenas prácticas participativas por el Observatorio Internacional de la Democracia Participativa.
} 


\section{Conclusiones: logros y retos}

El concepto de shrinking city se ha demostrado múltiple en sus causas y aplicaciones a diferentes espacios. Las estrategias de revitalización, por lo tanto, deben adaptarse a esta heterogeneidad de situaciones, siendo el papel de los actores locales en ellas una de las claves de su éxito. En este artículo se ha propuesto una interpretación de las estrategias de revitalización y de la desigual evolución de las ciudades en declive en base al objetivo que perseguían las estrategias y a los factores que existían o se activaban para ello. En dicha interpretación, la trayectoria de desarrollo seguida por las ciudades condicionaría su propia evolución futura, en sintonía con las propuestas de la geografía evolutiva. El resultado es un proceso de desarrollo acumulativo que se va modelando a lo largo del tiempo, pudiendo fortalecer la capacidad de reacción ante nuevas crisis o incidiendo en nuevas debilidades o bloqueos internos.

El análisis de los casos de estudio presentados ha mostrado dos ejemplos de crecimiento intenso durante varias décadas a partir de una rápida industrialización. Sin embargo, la evolución seguida por ambas ciudades tras la crisis de sus industrias ha sido desigual. Mientras Avilés ha sido capaz de revertir parcialmente su declive demográfico, económico y social, Langreo ha encontrado dificultades para mantener su población o mejorar su calidad de vida. Las causas de esto pueden hallarse en las respuestas políticas dadas al declive urbano y en el desigual papel de los actores locales en ellas.

En este sentido, mientras que en Langreo apenas se ha consensuado el modelo de desarrollo que se quería para la ciudad, generándose bloqueos internos y disminuyendo progresivamente la capacidad de concertación, en Avilés se ha avanzado hacia una «cultura de la concertación» encabezada por el Ayuntamiento de la ciudad, pero en la que otros actores también han tenido voz. De este modo, las estrategias de Langreo se han volcado a la reindustrialización de la ciudad, habiéndose subordinado el resto de iniciativas a este fin, lo que ha llevado a problemas entre distintos actores y, a la larga, ha reducido su operatividad. Por el contrario, en
Avilés se ha buscado armonizar crecimiento económico y planificación urbana, a través de inversiones en la imagen de la ciudad y de la reconversión de parte de los antiguos terrenos de ENSIDESA en equipamientos (mejorando su capacidad de resiliencia). Esto, aunque no ha evitado la aparición de algunos problemas, ha canalizado el potencial conflicto hacia la colaboración y la negociación. En definitiva, el análisis de estos dos casos de ciudades en declive ha demostrado el peso de los actores locales en las estrategias de revitalización y su influencia sobre la evolución de las ciudades más allá de la existencia de un contexto común, en este caso dominado por la reconversión económica.

\section{Referencias bibliográficas}

ALONSO, J.L. y MÉNDEZ, R. Innovación, pequeña empresa y desarrollo local en España. Madrid: Thomson Civitas. 2000.

AUDIRAC, I. \& ARROYO, J. (editors). Shrinking cities South/North. Guadalajara: Universiad de Guadalajara. 2010.

AYDALOT, P. El declive urbano y sus relaciones con la población y el empleo. Estudios territoriales, 1987, № 24, p. 15-32.

BAXTER, J. \& EYLES, J. Evaluating qualitative research in social geography: establishing 'rigour' in interview analysis. Transactions of the Institute of British Geographers, 1997, Vol. 22, No 4, p. 505-525.

BIERNACKI, P. \& WALDORF, D. Snowball sampling. Problems and techniques of chain referral sampling. Sociological methods and research, 1981, Vol. 10, № 2, p. 141-163.

BIRG, H. Demographic aging In: OSWALT, P. Shrinking Cities. Ostfildern: Hatje Cantz Publishers, 2006, p. 100-119.

BONTJE, M. Facing the challenge of Shrinking cities in East Germany: The case of Leipzig. Geojournal, 2004, Vol. 61, № 1, p. 13-21.

CAMAGNI, R. \& MAILLAT, D. (editors). Milieux innovateurs. Théorie et politiques. Paris: Economica, 2006. 
CASTELLS, M. y BORJA, J. Local y global. La gestión de las ciudades en la era de la información. Madrid: Taurus, 2000.

COOKE, P. \& SIMMIE, J. Knowledge, Innovation and the Competitiveness of Cities. In: BUCK, N. Changing Cities: Rethinking Urban Competitiveness, Cohesion and Governance. New York: Palgrave MacMillan, 2005, p. $97-111$.

CRANG, M. Qualitative methods: The new orthodoxy? Progress in Human Geograpny, 2002, Vol. 26, № 5, p. 647-655.

FLORIDA, R. The rise of the creative class and how it's transforming work, leisure, community and everyday life. New York: Basic Books, 2002.

FOL, S. \& CUNNINGHAM-SABOT, E. 'Declin urbain' et Shrinking Cities: une evaluation critique des approches de la décroissance urbaine. Annales de Géographie, 2010, $\mathrm{N}^{\circ} 674$, p. 359-383.

FUJI, Y. Shrinkage in Japan. In: OSWALT, P. Shrinking Cities. Ostfildern: Hatje Cantz Publishers, 2006, p. 86-99.

GONZÁLEZ, S. Scalar Narratives in Bilbao: A Cultural Politics of Scales Approach to the Study of Urban Policy. International Journal of Urban and Regional Research, 2006, Vol. 30, No 4, p. 836-857.

GRABHER, G. The weakness of strong ties: the 'lock-in' of regional development in the Ruhr area. In: GRABHER, G. The Embedded Firm: On the Socio-Economics of Industrial Networks. London: Routledge, 1993, p. 255-277.

GROSSMANN, K.; HAASE, A.; RINK, D. \& STEINFÜHRER, A. Urban Shrinkage in East Central Europe? Benefits and Limits of a Cross-National Transfer of Research Approaches. In: NOVAK, M. \& NOWOSIELSKİ, M. Declining Cities/Developing Cities: Polish and German Perspectives. Poznan: Instytut Zachodni, 2008, p. 77-99.

GUASCH, A.M. y ZULAIKA, J. Aprendiendo del Guggenheim Bilbao. Barcelona: Akal, 2007.
HAMDOUCH, A.; DEPRET, M.-H. \& TANGUY, C. Mondialisation et résilience des territoires: Trajectoires, dynamiques d'acteurs et expériences. Montréal: Presses de l'Université du Québec, 2012.

KOOIMAN, J. Governing as governance. London: Sage, 2003.

LUCY, W. \& PHILLIPS, D. Confronting suburban decline. Washington, D.C.: Island Press, 2000.

MARTIN, R. \& SUNLEY, P. Path dependence and regional economic revolution. Journal of Economic Geography, 2006, Vol. 6, No 4, p. 395-437.

MARTÍNEZ-FERNÁNDEZ, C.; AUDIRAC, I.; FOL, S. \& CUNNINGHAM-SABOT, E. Shrinking cities: Urban challenges of Globalisation. International Journal of Urban and Regional Research, 2012, Vol. 36, № 2, p. 213-225.

MÉNDEZ, R. (director). Estrategias de innovación industrial y desarrollo económico en las ciudades intermedias de España. Madrid: Fundación BBVA, 2010.

MÉNDEZ, R. Ciudades y metáforas: sobre el concepto de resiliencia urbana. Ciudad y territorio: estudios territoriales, 2012, № 172 , p. 215-232.

MUSTERD, S. \& MURIE, A. (editors) Making competitive cities. Oxford: Blackwell, 2010 .

OSWALT, P. (editor). Shrinking Cities. Ostfildern-Ruit: Hatje Cantz, 2006.

OSWALT, P. \& RIENIETS, T. Atlas of Shrinking cities. Ostfildern: Hatje Cantz Publishers, 2006.

PECK, J. Struggling with the creative class. International Journal of Urban and Regional Research, 2005, Vol. 76, № 6, p. 77-91.

PIKE, A.; DAWLEY, S. \& TOMANEY, J. Resilience, adaptation and adaptability. Cambridge Journal of Regions, Economy and Society, 2010, Vol. 3, No 1, p. 59-70. 
PINSON, G. Gouverner la ville par projet: Urbanisme et gouvernance des villes européennes. Paris: Les Presses de Sciences Po, 2009.

POLÈSE, M. The resilient city: on the determinants of successful urban economies. University of Québec: Centre Urbanisation, Culture Société, INRS, 2010.

PRADA, J. Desarrollo, patrimonio y políticas de revitalización en ciudades intermedias de especialización minero-industrial. El caso de Langreo (Asturias). Oviedo: Consejo Económico y Social de Asturias, 2011.

PRECEDO, A.; OROSA, J.J. y MÍGUEZ, A. De la planificación estratégica al marketing urbano: hacia la ciudad inmaterial. EURE, 2010, Vol. 36, No 108, p. 5-28.

SÁNCHEZ, S.; MÉNDEZ, R. y PRADA, J. Avilés, entre el declive y la revitalización: ¿en la génesis de un nuevo modelo de desarrollo? Boletín de la Asociación de Geógrafos Españoles, 2012, Nº6, p. 327-353.
SCOTT, A.J. Creative cities: conceptual issues and policy questions. Journal of Urban Affairs, 2006, Vol. 28, № 1, p. 1-17.

SIMMIE, J. Innovation and space: A critical review of the literature. Regional Studies, 2005, Vol. 39, Nº 6, p. 789-804.

SIMMIE, J. \& CARPENTER, J. Path dependence and the evolution of city regional economies. London: NESTA, 2008.

WALKER, R. The geography of production. In: SHEPPARD, E. y BARNES, T. A Companion to Economic Geography. Oxford: Blackwell, 2001, p. 113-132. 
